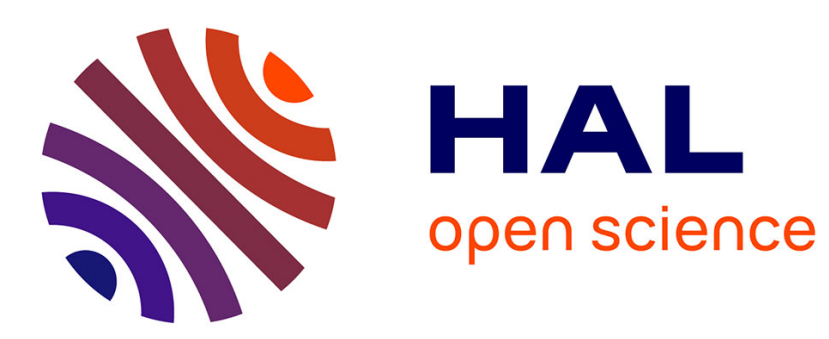

\title{
Controller Synthesis for Nonlinear Systems with Reachability Specifications Using Monotonicity
}

\author{
Vladimir Sinyakov, Antoine Girard
}

\section{To cite this version:}

Vladimir Sinyakov, Antoine Girard. Controller Synthesis for Nonlinear Systems with Reachability Specifications Using Monotonicity. IEEE Conference on Decision and Control, 2019, Nice, France. 10.1109/CDC40024.2019.9029740 . hal-02286407

\section{HAL Id: hal-02286407 https://hal.science/hal-02286407}

Submitted on 13 Sep 2019

HAL is a multi-disciplinary open access archive for the deposit and dissemination of scientific research documents, whether they are published or not. The documents may come from teaching and research institutions in France or abroad, or from public or private research centers.
L'archive ouverte pluridisciplinaire HAL, est destinée au dépôt et à la diffusion de documents scientifiques de niveau recherche, publiés ou non, émanant des établissements d'enseignement et de recherche français ou étrangers, des laboratoires publics ou privés. 


\title{
Controller Synthesis for Nonlinear Systems with Reachability Specifications Using Monotonicity
}

\author{
Vladimir Sinyakov and Antoine Girard
}

\begin{abstract}
In this paper, we consider a control synthesis problem for a continuous-time nonlinear system. The problem under consideration consists in driving the state of the system to some target interval at a given time instant. We propose a solution based on candidate under-approximations of the backward reachable sets using multi-dimensional intervals. We show that a suitable controller can be designed by enforcing a monotonicity property of the closed-loop system on these intervals. For this purpose, we utilize the monotonicity conditions for nonlinear systems with inputs in the infinitesimal form. From these differential inequalities on the control strategy, we design some particular controllers which are time-varying, linear with respect to the state. The approach is illustrated by two examples.
\end{abstract}

\section{INTRODUCTION}

This paper is concerned with the control problem of steering a nonlinear system to a specified target set at a given time instant. The system under consideration is of general nonlinear time-varying type with time-varying uncertainty. The proposed solution strategy is constructed using candidate multi-dimensional interval under-approximations of the backward reachable (i.e. co-reachable) sets. A suitable controller, which keeps the trajectories inside these intervals, can be designed by enforcing a monotonicity property for the closed-loop system.

There exist several different approaches to control synthesis problem in the aforementioned setting. Our approach belongs to the class of methods which do not require state space discretization and do not directly approximate the righthand side [1], [2], [3], [4]. Those methods usually consider simple-shaped regions like ellipsoids or parallelotops as basic under-approximations of the backward reachable sets. From these under-approximations, one can usually construct the corresponding controller (see, e.g. [5]). Such methods can yield families of approximations which may be combined into one covering a larger part of the actual co-reachable sets. Our paper follows this paradigm as well. Multi-dimensional intervals are obviously a particular case of parallelotops. However, we believe that the class of systems, to which our approach is applicable, and the proposed solution based on monotonicity, are different from what is present in the literature.

V. Sinyakov and A. Girard are with the Laboratoire des Signaux et Systèmes (L2S), CNRS, CentraleSupélec, Université Paris-Sud, Université, Paris-Saclay. 3, rue Joliot-Curie, 91192 Gif-sur-Yvette, cedex, France. email: vladimir.sinyakovel2s.centralesupelec.fr

This project has received funding from the European Research Council (ERC) under the European Union's Horizon 2020 research and innovation programme (grant agreement No 725144).
This work is motivated by symbolic control, a control design methodology where discrete abstractions (also called symbolic models) are used to enforce complex behaviors of continuous dynamical systems [6], [7]. In symbolic control, one of the methods for constructing a symbolic model from a continuous-time control system is to associate symbolic states with elements of a partition of the state space and to define transitions from one state to another if and only if there is a controller steering every trajectory initiating from the region associated with the first state to the one associated with the second state (see e.g. [8], [9]). The problem of polytope-to-polytope control for nonlinear systems in relation with symbolic control has also been studied using algebraic properties of multi-affine and polynomial vector fields in [10], [11], [12], or a combination of linear approximations with robust control in [13].

Our approach is based on a monotonicity property. Intuitively, monotone systems are systems that preserve some partial ordering on their trajectories. The notion of monotone systems with inputs was introduced in [14] where the definition depends on a particular partial order under consideration. In this paper, however, we focus on monotonicity with respect to a specific partial order on vector spaces given by less or equal relation between the components. In Section III, we establish sufficient conditions for monotonicity for a nonlinear time-varying system with inputs. Similar differential conditions are known for time-invariant systems with inputs [14] and time-varying systems without inputs (e.g. [15]). However, the authors are not aware of a previous result with both features.

In Section IV, we use the conditions for monotonicity to derive sufficient conditions on the controller to solve our reachability problem (Proposition 2). In Subsection IV.A, we then consider a class of monotone nonlinear control systems for which we verify using the obtained results that a particular control strategy solves the problem and is maximal in some sense. For that class of systems, the approach can be seen as an extension of [16] where a robust controlled invariance problem was considered, which is essentially a problem of controlling a multi-dimensional interval into itself. Next, in Subsection IV.B we consider a more general class of (not necessarily monotone) nonlinear systems and propose a solution based on time-varying linear feedback control strategies. Finally, in Subsection IV.C we discuss how to construct a hybrid controller applicable in a larger region from several control strategies obtained for different multi-dimensional intervals. 
The paper ends with two examples illustrating the approach. In the first example we construct one of the maximal by inclusion multi-dimensional intervals on which the reachability problem has a solution. The second example shows the application of our approach to an autonomous vehicle control problem. This example illustrates the intervalto-interval control synthesis using a number of elementary interval approximations.

Notations: For $x \in \mathbb{R}^{n},\|x\|_{\infty}=\max _{i}\left|x_{i}\right|$ is the infinity norm. Let $\operatorname{diag}(x)$ denote a diagonal matrix with elements of $x \in \mathbb{R}^{n}$ on the diagonal. Given vectors $x, x^{\prime} \in \mathbb{R}^{n}, x \preceq x^{\prime}$ stands for $x_{i} \leq x_{i}^{\prime}$ for all $i=1, \ldots, n$. Using this partial order, we define multi-dimensional interval sets as follows: for $\underline{x}, \bar{x} \in \mathbb{R}^{n},[\underline{x}, \bar{x}]=\{x \mid x \succeq \underline{x}, x \preceq \bar{x}\}$. Similarly, given matrices $M, M^{\prime}$ of the same dimensions, $M \preceq M^{\prime}$ stands for $M_{i, j} \leq M_{i, j}^{\prime}$ for all $i, j .2^{X}$ denotes the set of all subsets of $X$. For a set-valued map $W:[0, T] \longrightarrow 2^{\mathbb{R}^{n} w}$, the space of all Lebesgue measurable functions $w(\cdot)$ on $[0, T]$ such that $w(t) \in W(t)$ a.e. is denoted by $L^{\infty}([0, T], W(\cdot))$. For a set-valued map $Z:[0, T] \longrightarrow 2^{\mathbb{R}^{n_{x}}}$, we denote the set of all pairs $(t, x) \in[0, T] \times \mathbb{R}^{n_{x}}$ such that $x \in Z(t)$ by $\operatorname{graph}(Z)$. Given a function $h: A \longrightarrow \mathbb{R}$, we denote by Arg $\min _{a \in A} h(a)$ the set of all minimizers $\left\{a^{*} \in A \mid h\left(a^{*}\right)=\min _{a \in A} h(a)\right\}$.

\section{PROBLEM FORMULATION}

Consider a nonlinear system of the form:

$$
\dot{x}=f(t, x, u, w), \quad u \in \mathbb{R}^{n_{u}}, \quad w \in W(t) \subseteq \mathbb{R}^{n_{w}} .
$$

Here $u$ is the control and $w$ is the disturbance. The problem under consideration is to synthesize a control strategy $u$ : $[0, T] \times \mathbb{R}^{n_{x}} \rightarrow \mathbb{R}^{n_{u}}$, which takes values in $U \subseteq \mathbb{R}^{n_{u}}$ and steers the system to the target set $X^{1} \subseteq \mathbb{R}^{n_{x}}$ at time $T>0$, for any disturbance $w$.

We assume that the vector field $f$ is continuous in $(t, x, u, w)$, continuously differentiable in $(x, u, w)$, Lipschitz in $(x, u)$. In particular, suppose we have

$$
\|f(t, x, u, w)-f(t, y, u, w)\|_{\infty} \leq L\|x-y\|_{\infty} .
$$

A control strategy $u:[0, T] \times \mathbb{R}^{n_{x}} \rightarrow \mathbb{R}^{n_{u}}$ is admissible if $u$ is continuous in $(t, x)$, continuously differentiable and Lipschitz in $x \in \mathbb{R}^{n_{x}}$. We assume that the set of all possible realizations of the disturbance $w$ is $L^{\infty}([0, T], W(\cdot))$ where the map $W(\cdot)$ is continuous.

Now let $u$ be an admissible control strategy. Consider the closed-loop system

$$
\dot{x}=f(t, x, u(t, x), w)=F(t, x, w), \quad w \in W(t) .
$$

Due to our assumptions, for all initial positions $\left(t_{0}, x^{0}\right) \in$ $[0, T) \times \mathbb{R}^{n_{x}}$ and all admissible disturbances $w \in$ $L^{\infty}([0, T], W(\cdot))$, the solution of this system exists on the whole interval $[0, T]$ and is unique. Let us denote this solution as $\mathbf{x}\left(\cdot ; t_{0}, x, w\right)$.

Next, we give the definition of strong invariance.

Definition 1: A set-valued map $Z:[0, T] \longrightarrow 2^{\mathbb{R}^{n_{x}}}$ is strongly invariant with respect to $F$ if for all $0 \leq t_{1}<t_{2} \leq$
$T$, for all $x \in Z\left(t_{1}\right)$ and all $w \in L^{\infty}([0, T], W)$ the solution $\mathbf{x}\left(\cdot ; t_{1}, x, w\right)$ exists on $\left[t_{1}, t_{2}\right]$ and the following inclusion holds: $\mathbf{x}\left(t_{2} ; t_{1}, x, w\right) \in Z\left(t_{2}\right)$.

Formally the main problem we consider in this paper is formulated as follows.

Problem 1: Design an admissible control strategy $u$ : $[0, T] \times \mathbb{R}^{n_{x}} \rightarrow \mathbb{R}^{n_{u}}$ and a set-valued map $X^{-}:[0, T] \longrightarrow$ $2^{\mathbb{R}^{n_{x}}}$, strongly invariant with respect to $F$, such that

$$
u(t, x) \in U \subseteq \mathbb{R}^{n_{u}} \quad \text { for all } \quad(t, x) \in \operatorname{graph}\left(X^{-}\right),
$$

and for every position $\left(t_{0}, x^{0}\right) \in \operatorname{graph}\left(X^{-}\right)$, the trajectory of the closed-loop system (3) which starts from this position reaches the target set at time $T: \mathbf{x}\left(T ; t_{0}, x^{0}, w\right) \in X^{1}$ for all $w \in L^{\infty}([0, T], W(\cdot))$.

We call the set $X^{-}(t)$ the controllable set at time $t$. We would like to emphasize that if $X^{-}$is strongly invariant with respect to $F$ and such that $X^{-}(T)=X^{1}$, then by Definition 1 , it follows that $\mathbf{x}\left(T ; t_{0}, x^{0}, w\right) \in X^{1}$, for all $\left(t_{0}, x^{0}\right) \in \operatorname{graph}\left(X^{-}\right)$, for all $w \in L^{\infty}([0, T], W(\cdot))$.

For the following discussion let us introduce some additional related notion.

Definition 2: The set of all states $x$ for which there exist an initial state $x^{0} \in X^{0}$ and an admissible input $w(\cdot)$ such that $\mathbf{x}\left(t ; 0, x^{0}, w\right)=x$ is called the reachable set of system (3) and is denoted $X_{r}\left(t ; 0, X^{0}\right)$.

\section{SUFFICIENT CONDITION FOR MONOTONICITY}

Consider a nonlinear system of the form

$$
\dot{x}=F(t, x, w), \quad w \in W(t) .
$$

Here $W(t)=[\underline{w}(t), \bar{w}(t)]$ where $\underline{w}(\cdot)$ and $\bar{w}(\cdot)$ are continuous. Let $F$ be continuous in $(t, x, w)$, continuously differentiable in $x$ and in $w$ and Lipschitz in $x$. Let $Z$ be a set-valued map, strongly invariant with respect to $F$.

Definition 3: The system (4) is monotone in $\operatorname{graph}(Z)$ if for all $0 \leq t_{1}<t_{2} \leq T$, for all $x, x^{\prime} \in Z\left(t_{1}\right)$ for all $w, w^{\prime} \in L^{\infty}\left(\left[t_{1}, t_{2}\right], W(\cdot)\right)$

$$
\begin{aligned}
& x \preceq x^{\prime}, \text { and } \forall t \in\left[t_{1}, t_{2}\right], w(t) \preceq w^{\prime}(t) \\
\Rightarrow \quad & \forall t \in\left[t_{1}, t_{2}\right], \mathbf{x}\left(t ; t_{1}, x, w\right) \preceq \mathbf{x}\left(t ; t_{1}, x^{\prime}, w^{\prime}\right) .
\end{aligned}
$$

If $Z(t)=\mathbb{R}^{n_{x}}$ for $t \in[0, T]$ the system is simply called monotone.

Now consider $Z(t)=[\underline{x}(t), \bar{x}(t)]$ where $\bar{x}(\cdot)$ and $\underline{x}(\cdot)$ solve the following system of equations:

$$
\begin{array}{ll}
\dot{\bar{x}}(t)=F(t, \bar{x}(t), \bar{w}(t)), & \bar{x}(0)=\bar{x}^{0}, \\
\underline{\dot{x}}(t)=F(t, \underline{x}(t), \underline{w}(t)), & \underline{x}(0)=\underline{x}^{0} .
\end{array}
$$

Under our assumptions, the solution to these equations exists on the whole time interval $[0, T]$. The main result of this section is the following. 
Proposition 1: Consider system (4), initial value problem (6), (7) and let the following conditions hold for all $(t, x)$ in some neighborhood of $\operatorname{graph}(Z)$ and all $w \in W(t)$ :

$$
\begin{gathered}
\frac{\partial F_{i}}{\partial x_{j}}(t, x, w) \geq 0, \quad \forall i \neq j, \\
\frac{\partial F_{i}}{\partial w_{k}}(t, x, w) \geq 0, \quad \forall i, k .
\end{gathered}
$$

Then $Z(t) \neq \emptyset$, for all $t \in[0, T]$ and $Z$ is strongly invariant with respect to $F$.

Remark 1: In the following proof we, in fact, only use the continuity and monotonicity of $F_{i}$ with respect to the corresponding components of the state space vector and the inputs. The monotonicity condition in the differential form above may be easier to use in the synthesis problem.

Proof: First, let us establish the claim of the proposition in the case when conditions (8), (9) hold for all $x \in \mathbb{R}^{n_{x}}$.

To prove it we utilize the dynamic optimization approach. Let us define the value function:

$$
\begin{gathered}
V(t, x)=\min _{w \in L^{\infty}([0, t], W(\cdot))} \sigma(\mathbf{x}(0 ; t, x, w))= \\
\min _{w \in L^{\infty}([0, t], W(\cdot))} \max _{i} \max \left\{\mathbf{x}_{i}(0 ; t, x, w)-\bar{x}_{i}^{0},\right. \\
\left.\underline{x}_{i}^{0}-\mathbf{x}_{i}(0 ; t, x, w), 0\right\} .
\end{gathered}
$$

On one hand, the reachable set at time $t$ satisfies the relation [17]:

$$
X_{r}\left(t ; 0, X^{0}\right)=\{x \mid V(t, x) \leq 0\}, \quad X^{0}=\left[\underline{x}^{0}, \bar{x}^{0}\right] .
$$

On the other hand, $V$ is the viscosity solution of the Hamilton-Jacobi-Bellman equation (for instance, see [18])

$$
V_{t}+\max _{w \in W(t)}\left\langle V_{x}, F(t, x, w)\right\rangle=0, \quad V(0, x)=\sigma(x) .
$$

Consider now a function of the form

$$
V^{-}(t, x)=e^{-L t} \max _{i} \max \left\{x_{i}-\bar{x}_{i}(t), \underline{x}_{i}(t)-x_{i}, 0\right\} .
$$

For inequality

$$
V^{-}(t, x) \leq V(t, x)
$$

to hold it is sufficient for $V^{-}$to be a subsolution of equation (11):

$$
\begin{gathered}
q+\max _{w \in W(t)}\langle p, F(t, x, w)\rangle \leq 0,(q, p) \in D^{+} V^{-}(t, x) \\
V^{-}(0, x)=\sigma(x), \quad x \in \mathbb{R}^{n_{x}}
\end{gathered}
$$

Since $V^{-}$is convex in $x$, its superdifferential $D^{+} V^{-}(t, x)$ is either empty or singleton for all $(t, x)$. Therefore, it is sufficient for (14) to hold at every point of differentiability of $V^{-}$.

Let us consider the case when $V^{-}(t, x) e^{L t}=x_{i}-\bar{x}_{i}(t)$ :

$$
-L V^{-}(t, x) e^{L t}-\dot{\bar{x}}_{i}(t)+\max _{w \in W(t)} F_{i}(t, x, w) \leq 0
$$

Since $F_{i}$ is monotone in each $w_{k}$, we have

$$
\dot{\bar{x}}_{i}(t) \geq F_{i}(t, x, \bar{w}(t))-L V^{-}(t, x) e^{L t} .
$$

Let

$$
\bar{\xi}_{j}(t, x)=\left\{\begin{aligned}
x_{j}, & x_{j} \leq \bar{x}_{j}(t), \quad, \quad j=1, \ldots, n . \\
\bar{x}_{j}(t), & x_{j}>\bar{x}_{j} .
\end{aligned}\right.
$$

Then we estimate

$$
\begin{gathered}
F_{i}(t, x, \bar{w}(t))-L V^{-}(t, x) e^{L t} \leq \\
F_{i}(t, \bar{\xi}(t, x), \bar{w}(t))+\left[L\|x-\bar{\xi}(t, x)\|_{\infty}-L V^{-}(t, x) e^{L t}\right] .
\end{gathered}
$$

Here we utilized (2). One may observe that the expression in the square brackets is equal to zero. Note that in the considered case $\bar{\xi}_{i}(t, x)=\bar{x}_{i}(t)$. Using monotonicity of $F_{i}$ with respect to $x_{j}$ for $i \neq j$ we obtain the relation:

$$
\dot{\bar{x}}_{i}(t) \geq F_{i}(t, \bar{x}(t), \bar{w}(t)) \text {. }
$$

Similar reasoning in the case when $V^{-}(t, x) e^{L t}=\underline{x}_{i}(t)-$ $x_{i}$ gives us the differential inequality

$$
\underline{\dot{x}}_{i}(t) \leq F_{i}(t, \underline{x}(t), \underline{w}(t)) .
$$

Thus, for (14) and (15) to hold for all $(t, x) \in[0, T] \times \mathbb{R}^{n_{x}}$ it is sufficient that

$$
\begin{array}{ll}
\dot{\bar{x}}_{i}(t)=F_{i}(t, \bar{x}(t), \bar{w}(t)), & \bar{x}(0)=\bar{x}^{0}, \\
\dot{\dot{x}}_{i}(t)=F_{i}(t, \underline{x}(t), \underline{w}(t)), & \underline{x}(0)=\underline{x}^{0}
\end{array}
$$

which indeed hold. Therefore, a function $V^{-}$given by (12) is well-defined on $[0, T] \times \mathbb{R}^{n_{x}}$ and satisfies inequality (13). Observe from the definition of the value function that

$$
V(t, \bar{x}(t))=V(t, \underline{x}(t))=0 .
$$

Therefore,

$$
V^{-}(t, \bar{x}(t))=V^{-}(t, \underline{x}(t))=0 .
$$

But this is only possible if $\underline{x}(t) \preceq \bar{x}(t)$. Thus, $Z(t) \neq \emptyset$.

Now from (10) and (13) we have the over-approximation:

$$
X_{r}\left(t ; 0, X^{0}\right) \subseteq Z(t) \equiv[\underline{x}(t), \bar{x}(t)] .
$$

From this relation it follows that for every $x^{0} \in X^{0}$ and every $w \in L^{\infty}([0, T], W)$ the solution $\mathbf{x}\left(\cdot ; 0, x^{0}, w\right)$ exists on $[0, T]$. Moreover, repeating the whole argument for $\left(t_{1}, Z\left(t_{1}\right)\right)$ instead of $\left(0, X^{0}\right)$ we obtain the relation:

$$
X_{r}\left(t ; t_{1}, Z\left(t_{1}\right)\right) \subseteq Z(t) \equiv[\underline{x}(t), \bar{x}(t)] .
$$

Therefore, from the definition of the reachable set we get the strong invariance of $Z$.

Observe now that the property $Z(t) \neq \emptyset$ depend on values of $F$ in an arbitrary small neighborhood of $\{(t, \bar{x}(t), \bar{w}(t)), t \in[0, T]\}$ and $\{(t, \underline{x}(t), \underline{w}(t)), t \in[0, T]\}$. In turn, the property of $Z$ being strongly invariant depend on values of $F$ in an arbitrary small neighborhood of $\operatorname{graph}(Z)$. This observation completes the proof of the proposition.

Corollary 1: The system (4) is monotone in $\operatorname{graph}(Z)$. If in Proposition 1 conditions (8), (9) hold for all $x \in \mathbb{R}^{n_{x}}$ then the system is monotone.

Proof: Consider $x, x^{\prime} \in Z\left(t_{1}\right), x \preceq x^{\prime}$ and some $w, w^{\prime} \in L^{\infty}\left(\left[t_{1}, t_{2}\right], W\right), w(t) \preceq w^{\prime}(t), t \in\left[t_{1}, t_{2}\right]$. Since $Z$ is strongly invariant and $\left[x, x^{\prime}\right] \subseteq Z\left(t_{1}\right)$, al1 trajectories starting from $\left[x, x^{\prime}\right]$ at time $t_{1}$ lie inside 
$\operatorname{graph}(Z)$. Then the assumptions of Proposition 1 hold for $\left[\mathbf{x}\left(t ; t_{1}, x, w\right), \mathbf{x}\left(t, t_{1}, x^{\prime}, w^{\prime}\right)\right]$. Thus, this interval is nonempty for all $t \in\left[t_{1}, t_{2}\right]$ or, in other words, $\mathbf{x}\left(t ; t_{1}, x, w\right) \preceq$ $\mathbf{x}\left(t, t_{1}, x^{\prime}, w^{\prime}\right)$.

\section{CONTROL SYNTHESIS}

In this section, we derive the conditions for control strategies $u:[0, T] \times \mathbb{R}^{n_{x}} \rightarrow \mathbb{R}^{n_{u}}$ to solve the problem of steering system (1) into the target set $X^{1}=\left[\underline{x}^{1}, \bar{x}^{1}\right]$. We assume that $W(t)=[\underline{w}(t), \bar{w}(t)]$ as previously.

Consider two control inputs $u^{1}(\cdot), u^{2}(\cdot) \in L^{\infty}([0, T] ; U)$. Let $\bar{x}(\cdot)$ and $\underline{x}(\cdot)$ be the trajectories of system (1) with disturbances $\bar{w}(\cdot)$ and $\underline{w}(\cdot)$ correspondingly:

$$
\begin{array}{ll}
\dot{\bar{x}}(t)=f\left(t, \bar{x}(t), u^{1}(t), \bar{w}(t)\right), & \bar{x}(T)=\bar{x}^{1}, \\
\underline{\dot{x}}(t)=f\left(t, \underline{x}(t), u^{2}(t), \underline{w}(t)\right), & \underline{x}(T)=\underline{x}^{1} .
\end{array}
$$

Those equations are to be integrated backward in time on the interval $[0, T]$. We assume that $\underline{x}(t) \preceq \bar{x}(t)$, for all $t \in[0, T]$. In our construction we use the interval $[\underline{x}(t), \bar{x}(t)]$ as the controllable set $X^{-}(t)$.

Proposition 2: Consider a control strategy $u(t, x)$ which is continuous in $(t, x)$, continuously differentiable and Lipschitz in $x$ and such that $u(t, \bar{x}(t))=u^{1}(t)$ and $u(t, \underline{x}(t))=$ $u^{2}(t)$ where the functions $\bar{x}(\cdot)$ and $\underline{x}(\cdot)$ are defined by equations (16) and (17) respectively with $\underline{x}(t) \preceq \bar{x}(t)$, $t \in[0, T]$. Suppose that $u(t, x) \in U$ for all $t \in[0, T]$ and $x \in[\underline{x}(t), \bar{x}(t)]$. Let conditions

$$
\begin{gathered}
\frac{\partial f(t, x, u(t, x), w)}{\partial x}+\frac{\partial f(t, x, u(t, x), w)}{\partial u} \frac{\partial u(t, x)}{\partial x} \succeq \gamma I \\
\frac{\partial f(t, x, u(t, x), w)}{\partial w} \succeq 0
\end{gathered}
$$

be satisfied for all $t \in[0, T]$, for all $x$ in some neighborhood of $[\underline{x}(t), \bar{x}(t)]$, all $w \in W(t)$ and for some $\gamma \in \mathbb{R}$. Here $I$ is the identity matrix. Then $u$ and $X^{-}$given by $X^{-}(t)=$ $[\underline{x}(t), \bar{x}(t)]$ solve Problem 1 .

Proof: For this strategy to solve control synthesis problem of reaching the target set $X^{1}$, it is sufficient that the closed-loop system satisfies the conditions of Proposition 1 since $X^{-}(T)=X^{1}$. This is indeed the case since the conditions (18) and (19) coincide with (8) and (9) respectively written in a matrix form. It then follows that $X^{-}$is strongly invariant and $u$ enforces the monotonicity of the closed-loop system in $\operatorname{graph}\left(X^{-}\right)$.

\section{A. Special case}

Let us now consider a nonlinear system with the following special structure $\left(n_{u} \leq n_{x}\right)$ :

$$
\begin{array}{ll}
\dot{x}_{i}=f_{i}\left(t, x, u_{i}, w\right), & 1 \leq i \leq n_{u}, \\
\dot{x}_{i}=f_{i}(t, x, w), & n_{u}<i \leq n_{x} .
\end{array}
$$

Here $u \in U=[\underline{u}, \bar{u}]$ and $w \in W(t)$. Let us introduce some notations:

$$
\begin{array}{cl}
\hat{x}(t)=\frac{1}{2}(\bar{x}(t)+\underline{x}(t)), & \tilde{x}(t)=\frac{1}{2}(\bar{x}(t)-\underline{x}(t)), \\
\hat{u}=\frac{1}{2}(\bar{u}+\underline{u}), & \tilde{u}=\frac{1}{2}(\bar{u}-\underline{u}) .
\end{array}
$$

Proposition 3: Let the monotonicity conditions

$$
\frac{\partial f_{i}}{\partial x_{j}} \geq 0, \quad \frac{\partial f_{i}}{\partial u_{i}} \geq 0, \quad \frac{\partial f_{i}}{\partial w_{k}} \geq 0
$$

hold for all $1 \leq i, j \leq n_{x}, 1 \leq k \leq n_{w}, i \neq j$, for all $t \in[0, T]$, for all $u \in U$ and all $w \in W(t)$.

Consider an interval $X^{-}(t)=[\underline{x}(t), \bar{x}(t)]$ defined by the equations

$$
\begin{aligned}
\dot{\bar{x}}_{i}(t) & =f_{i}\left(t, \bar{x}(t), \underline{u}_{i}, \bar{w}(t)\right), & & 1 \leq i \leq n_{u}, \\
\dot{\bar{x}}_{i}(t) & =f_{i}(t, \bar{x}(t), \bar{w}(t)), & & n_{u}<i \leq n_{x}, \\
\dot{x}_{i}(t) & =f_{i}\left(t, \underline{x}(t), \bar{u}_{i}, \underline{w}(t)\right), & & 1 \leq i \leq n_{u}, \\
\dot{\dot{x}}_{i}(t) & =f_{i}(t, \underline{x}(t), \underline{w}(t)), & & n_{u}<i \leq n_{x} .
\end{aligned}
$$

with the terminal conditions

$$
\bar{x}(T)=\bar{x}^{1}, \quad \underline{x}(T)=\underline{x}^{1} .
$$

This is a maximal by inclusion interval which solves Problem 1 for some strategy $u(t, x)$ : every multi-dimensional interval $Z \supset X^{-}(t)$ contains uncontrollable states.

Proof: First, observe that the strategy $u^{*}$ defined by

$$
u_{i}^{*}(t, x)=\hat{u}_{i}-\frac{x_{i}-\hat{x}_{i}(t)}{\tilde{x}_{i}(t)} \tilde{u}_{i}
$$

solves Problem 1 for the controllable set $X^{-}(t)=$ $[\underline{x}(t), \bar{x}(t)]$. Indeed, conditions of Proposition 2 are clearly satisfied in this case.

Now let $u(\cdot)$ be an arbitrary control input from $L^{\infty}([0, T], U)$. Consider the following system

$$
\begin{array}{lll}
\dot{x}_{i}(t)=f_{i}\left(t, x(t), u_{i}(t), \bar{w}(t)\right), & & i=1, \ldots, n_{u}, \\
\dot{x}_{i}(t)=f_{i}(t, x(t), \bar{w}(t)), & & n_{u}<i \leq n_{x}
\end{array}
$$

with the initial condition

$$
x\left(t_{0}\right)=x^{0} .
$$

Assume that $x^{0} \succ \bar{x}\left(t_{0}\right)$. Then due to monotonicity of (20) and uniqueness of its solution, $x(T) \succ \bar{x}^{1}$. Therefore, the system is not controllable from $\left(t_{0}, x^{0}\right)$.

Similar reasoning for the case $x^{0} \prec \underline{x}^{1}$ completes the proof.

\section{B. Linear feedback case}

We build up on the previous case considering now more general linear feedback control strategies for system (1) with $U=[\underline{u}, \bar{u}]$. Let $\Gamma(t)=\left(\gamma_{k i}(t)\right)$ be an $n_{u} \times n_{x}$ matrix such that

$$
\sum_{i=1}^{n_{i}} \gamma_{k i}(t) \leq 1, \quad \gamma_{k i}(t) \geq 0, \quad \forall k .
$$

Then we may define a linear-feedback control strategy $u$ by the formulas:

$$
\begin{gathered}
u(t, x)=\hat{u}-\operatorname{diag}(\tilde{u}) \Gamma(t)[\operatorname{diag}(\tilde{x}(t))]^{-1}(x-\hat{x}(t)), \\
\dot{\bar{x}}(t)=f(t, \bar{x}(t), \hat{u}-\operatorname{diag}(\tilde{u}) \Gamma(t) e, \bar{w}(t)), \quad \bar{x}(T)=\bar{x}^{1}, \\
\underline{\dot{x}}(t)=f(t, \underline{x}(t), \hat{u}+\operatorname{diag}(\tilde{u}) \Gamma(t) e, \underline{w}(t)), \quad \underline{x}(T)=\underline{x}^{1}
\end{gathered}
$$

where $\hat{x}(t), \tilde{x}(t), \hat{u}$ and $\tilde{u}$ are the same as in (21) and $e=(1, \ldots, 1)^{T}$. If $\underline{x}_{i}(t)<\bar{x}_{i}(t)$ for all $1 \leq i \leq n_{x}$ and all $t \in[0, T]$ then this strategy is well-defined in the 
region graph $\left(X^{-}\right)$. Indeed, one may directly check that $u(t, x) \in[\underline{u}, \bar{u}]$ in this region. Controllers of this form (for parallelotopic regions) were considered in [3].

The monotonicity condition above gives us a relation for $\Gamma(t)$ :

$$
\begin{gathered}
\frac{\partial f(t, x, u(t, x), w)}{\partial x} \operatorname{diag}(\tilde{x}(t))- \\
\frac{\partial f(t, x, u(t, x), w)}{\partial u} \operatorname{diag}(\tilde{u}) \Gamma(t) \succeq \gamma I, \\
\forall t \in[0, T], \forall x \in \mathbb{R}^{n_{x}}, \forall w \in W(t)
\end{gathered}
$$

for some $\gamma \in \mathbb{R}$. The special case of the previous subsection corresponds to $\Gamma(t)=I_{n_{u} n_{x}}$.

Now suppose we have the following estimates

$$
\frac{\partial f(t, x, u, w)}{\partial x} \succeq A(t), \quad \frac{\partial f(t, x, u(t, x), w)}{\partial u} \preceq B(t) .
$$

Then it is sufficient for $\Gamma(t)$ to satisfy

$$
A(t) \operatorname{diag}(\tilde{x}(t)) \succeq B(t) \operatorname{diag}(\tilde{u}) \Gamma(t)+\gamma I .
$$

For example, if $f(t, x, u, w)=A(t, w) x+B(t, w) u+c(t, w)$ with $A(t, w) \in[\underline{A}, \bar{A}]$ and $B(t, w) \in[\underline{B}, \bar{B}]$ then we have the following condition:

$$
\underline{A} \operatorname{diag}(\tilde{x}(t)) \succeq \bar{B} \operatorname{diag}(\tilde{u}) \Gamma(t)+\gamma I .
$$

\section{Discontinuous control strategies}

Normally, a different choice of inputs $u^{1}$ and $u^{2}$ results in different interval sets $[\underline{x}(t), \bar{x}(t)]$. In general, among those sets there are maximal ones: the intervals which are not included into any other such intervals at some $t$. Computing different such intervals we may then construct a discontinuous controller for a larger controllable set as follows.

Let $u^{s}(t, x), s=1, \ldots, N$ be basic controllers for the controllable intervals $X_{s}^{-}(t)=\left[\underline{x}^{s}(t), \bar{x}^{s}(t)\right]$ and let $V_{s}^{-}(t, x)$ be the corresponding functions of type (12). Then $u(t, x)$ can be formally defined by

$$
\begin{gathered}
s_{*}(t, x)=\min \left[\operatorname{Arg} \min _{1 \leq s \leq N} V_{s}^{-}(t, x)\right] \\
\hat{x}^{s}(t)=\left(\bar{x}^{s}(t)+\underline{x}^{s}(t)\right) / 2, \tilde{x}^{s}(t)=\left(\bar{x}^{s}(t)-\underline{x}^{s}(t)\right) / 2 \\
u(t, x)=u^{s_{*}(t, x)}(t, x)
\end{gathered}
$$

with the corresponding controllable set

$$
X^{-}(t)=\bigcup_{1 \leq s \leq N} X_{s}^{-}(t) \text {. }
$$

Effectively, we chose the number $s$ such that $x\left(t_{0}\right) \in X_{s}^{-}\left(t_{0}\right)$ once and then use the corresponding control strategy $u^{s}(t, x)$.

The controller $u(t, x)$ above solves a version of Problem 1 where the admissible strategy is just Lebesgue measurable in $(t, x)$.

\section{EXAMPLES}

Example 1. Consider a two-dimensional nonlinear system:

$$
\begin{aligned}
& \dot{x}_{1}=u x_{1}\left(1+x_{2}\right)+w_{1}, \\
& \dot{x}_{2}=-\frac{1}{1+x_{1}^{2}}-\frac{2 x_{2}^{3}}{1+x_{2}^{2}}+w_{2} .
\end{aligned}
$$

Here $u \in[0,1]$ is the control, $w \in[-1,0] \times[-1,1]$ is the disturbance. This system is of the type (20) above. Let us

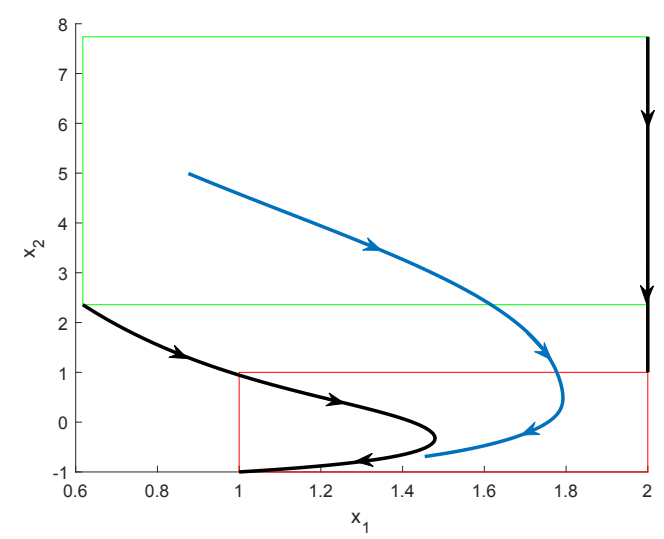

Fig. 1. Simulated trajectory of the closed-loop system.

compute the strategy and the corresponding controllable set of Proposition 3.

Figure 1 depicts the target set in red, $X^{-}(0)$ in green, $\bar{x}(\cdot)$ and $\underline{x}(\cdot)$ in black and a simulated trajectory $x(\cdot)$ of the closed-loop system in blue.

Example 2. Examples of monotone systems naturally arise in many applications. One such area is the control of autonomous vehicles. In [19], for instance, an efficient solution to the vehicle platooning problem, which is based on monotonicity of the system, was proposed.

Here we would like to consider the following takeover maneuvre problem. Taking the kinematic car model as a starting point we then perform an input transformation which gives the following nonlinear control system ([20], p. 371):

$$
\begin{aligned}
\dot{x} & =u_{1}, \\
\dot{y} & =u_{1} \tan \theta, \\
\dot{\theta} & =u_{1} \tan \phi, \\
\dot{\phi} & =u_{2} .
\end{aligned}
$$

Here $u_{1} \in[0,1], u_{2} \in[-1,1]$ are the controls. Variables $x$ and $y$ describe the position of the car, $\theta$ is the angle between the car body and $x$ axis, $\phi$ is the steering angle with respect to the car body. This model is applicable for small absolute values of $\theta$ and $\phi$. Therefore, we pose the following statespace constraints $|\theta| \leq \theta_{1},|\phi| \leq \phi_{1}<\pi / 2$.

In the following we only consider the last three equations of this system. Let $z=[y, \theta, \phi]^{T}$. The goal of control here is to steer the system from any state in

$$
X^{0}=[0.01,0.03] \times[-0.01,0.01] \times[-0.001,0.001]
$$

to some state in

$$
X^{1}=[-0.01,0.01] \times[-0.01,0.01] \times[-0.001,0.001] .
$$

Physically, if the road is aligned with the $x$ axis, this may correspond to a transition from one lane to another.

As we consider potential applications of the approach to the symbolic control framework, the regions $X^{0}$ and $X^{1}$ may, for instance, represent two states of a symbolic model. If using our approach we obtain a controllable set which includes $X^{0}$ then the symbolic model would have the corresponding transition between $X^{0}$ and $X^{1}$. 


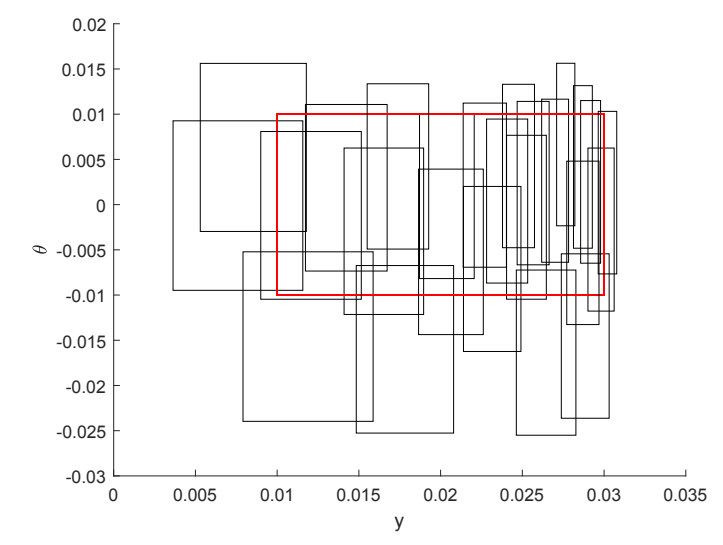

Fig. 2. Cover of the initial region $X^{0}$ by the controllable regions of different basic control strategies.

Proceeding to the solution, the monotonicity condition reads

$\left[\begin{array}{ccc}0 & u_{1} / \cos ^{2} \theta & 0 \\ 0 & 0 & u_{1} / \cos ^{2} \phi \\ 0 & 0 & 0\end{array}\right]+\left[\begin{array}{cc}\tan \theta & 0 \\ \tan \phi & 0 \\ 0 & 1\end{array}\right] \frac{\partial u(t, z)}{\partial z} \succeq \gamma I$.

Therefore, we obtain the relations:

$$
\begin{gathered}
\frac{\partial u_{1}(t, z)}{\partial \theta} \tan \theta+\frac{u_{1}}{\cos ^{2} \theta} \geq 0, \\
\frac{\partial u_{1}(t, z)}{\partial \phi} \tan \theta \geq 0, \quad \frac{\partial u_{1}(t, z)}{\partial y} \tan \phi \geq 0, \\
\frac{\partial u_{1}(t, z)}{\partial \phi} \tan \phi+\frac{u_{1}}{\cos ^{2} \phi} \geq 0, \\
\frac{\partial u_{2}(t, z)}{\partial y} \geq 0, \quad \frac{\partial u_{2}(t, z)}{\partial \theta} \geq 0 .
\end{gathered}
$$

Let $u^{1}(\cdot)$ and $u^{2}(\cdot)$ be arbitrary control inputs with $u_{1}^{1}(t)=u_{1}^{2}(t)$, let $\bar{z}(\cdot)=(\bar{y}(\cdot), \bar{\theta}(\cdot), \bar{\phi}(\cdot))^{T}$ and $\underline{z}(\cdot)=$ $(\underline{y}(\cdot), \underline{\theta}(\cdot), \underline{\phi}(\cdot))^{T}$ form the solution of the corresponding system (16), (17) and define a control strategy as follows:

$$
u(t, z)=\left[\begin{array}{c}
u_{1}(t) \\
\frac{1}{2}\left(u_{2}^{1}(t)+u_{2}^{2}(t)\right)-\frac{1}{2} \frac{\phi-\hat{\phi}(t)}{\tilde{\phi}(t)}\left(u_{2}^{1}(t)-u_{2}^{2}(t)\right)
\end{array}\right] .
$$

Here $\hat{\phi}(t)=\frac{1}{2}(\bar{\phi}(t)+\underline{\phi}(t))$ and $\tilde{\phi}(t)=\frac{1}{2}(\bar{\phi}(t)-\underline{\phi}(t))$. Then $u(t, z)$ is an admissible control strategy which satisfies the monotonicity condition and solves Problem 1 in $X^{-}(t)$.

Constructing a discontinuous control strategy as shown above we are able to solve the control problem from any state in $X^{0}$. In this construction we compute the trajectories of (16), (17) checking that the condition $\underline{z}(t) \preceq \bar{z}(t)$ holds for all $t \in[0, T]$.

Figure 2 depicts the controllable sets $X^{-}(0)$ for different basic control strategies (in black) which correspond to different control inputs $u^{1}(\cdot)$ and $u^{2}(\cdot)$. The initial state $X^{0}$ is given in red.

\section{CONCLUSION}

The considered approach gives a new solution to the control synthesis problem for a certain class of nonlinear control systems with uncertain parameters. The condition which must be satisfied by a control strategy ultimately is a system of linear partial differential inequalities. Although, finding a solution to it may be very difficult in general, we presented certain classes of systems and examples where it is possible. The last example illustrates interval-to-interval control synthesis which could be useful from the symbolic control perspective. We are planning to expand in this direction in our future work. We also plan to extend the results to the continuous-time analogue of so called mixedmonotone systems.

\section{REFERENCES}

[1] A. B. Kurzhanski and I. Valyi, Ellipsoidal Calculus for Estimation and Control. SCFA. Birkhäuser, Boston, 1995.

[2] J. K. Scott and P. I. Barton, "Bounds on the reachable sets of nonlinear control systems," Automatica, vol. 49, no. 1, pp. 93-100, Jan. 2013.

[3] E. K. Kostousova, "On control synthesis for uncertain differential systems using a polyhedral technique," in Large-Scale Scientific Computing, I. Lirkov, S. Margenov, and J. Waśniewski, Eds. Springer Berlin Heidelberg, 2014, pp. 98-106.

[4] V. V. Sinyakov, "Method for computing exterior and interior approximations to the reachability sets of bilinear differential systems," Differential Equations, vol. 51, no. 8, pp. 1097-1111, 2015.

[5] A. I. Subbotin, Generalized Solutions of First Order PDEs: The Dynamical Optimization Perspective. Birkhäuser Basel, 1995.

[6] P. Tabuada, Verification and Control of Hybrid Systems: a symbolic approach. Springer, 2008.

[7] C. Belta, B. Yordanov, and E. A. Gol, Formal Methods for DiscreteTime Dynamical Systems. Springer, 2017.

[8] P. E. Caines and Y. J. Wei, "Hierarchical hybrid control systems: A lattice theoretic formulation," IEEE Transactions on Automatic Control, vol. 43, no. 4, pp. 501-508, 1998.

[9] E. A. Gol, M. Lazar, and C. Belta, "Language-guided controller synthesis for linear systems," IEEE Transactions on Automatic Control, vol. 59, no. 5, pp. 1163-1176, 2014.

[10] C. Belta and L. C. Habets, "Controlling a class of nonlinear systems on rectangles," IEEE Transactions on Automatic Control, vol. 51, no. 11, pp. 1749-1759, 2006.

[11] M. A. Ben Sassi and A. Girard, "Control of polynomial dynamical systems on rectangles," in 2013 European Control Conference (ECC). IEEE, 2013, pp. 658-663.

[12] C. Sloth and R. Wisniewski, "Control to facet for polynomial systems," in Proceedings of the 17th international conference on Hybrid systems: computation and control. ACM, 2014, pp. 123-132.

[13] A. Girard and S. Martin, "Synthesis for constrained nonlinear systems using hybridization and robust controllers on simplices," IEEE Transactions on Automatic Control, vol. 57, no. 4, pp. 1046-1051, 2012.

[14] D. Angeli and E. D. Sontag, "Monotone control systems," IEEE Transactions on Automatic Control, vol. 48, no. 10, pp. 1684-1698, 2003.

[15] N. Ramdani, N. Meslem, and Y. Candau, "Computing reachable sets for uncertain nonlinear monotone systems," Nonlinear Analysis: Hybrid Systems, vol. 4, no. 2, pp. 263 - 278, 2010, iFAC World Congress 2008.

[16] P. J. Meyer, A. Girard, and E. Witrant, "Robust controlled invariance for monotone systems: application to ventilation regulation in buildings," Automatica, vol. 70, pp. 14-20, 2016.

[17] A. B. Kurzhanski and P. Varaiya, "Dynamic optimization for reachability problems," Journal of Optimization Theory and Applications, vol. 108, no. 2, pp. 227-251, 2001.

[18] W. H. Fleming and H. M. Soner, Controlled Markov Processes and Viscosity Solutions. Springer-Verlag, New York, 1995.

[19] A. Saoud, A. Girard, and L. Fribourg, "Contract based design of symbolic controllers for interconnected multiperiodic sampled-data systems," in 57th IEEE Conference on Decision and Control, 2018.

[20] R. M. Murray, S. S. Sastry, and L. Zexiang, A Mathematical Introduction to Robotic Manipulation. CRC Press, 1994. 